\title{
Effect of melatonin administration on subjective sleep quality in chronic obstructive pulmonary disease
}

\author{
D.M. Nunes ${ }^{1}$, R.M.S. Mota ${ }^{2}$, M.O. Machado ${ }^{3}$, E.D.B. Pereira ${ }^{3}$, V.M.S. de Bruin ${ }^{3}$ and \\ P.F.C. de Bruin ${ }^{3}$ \\ ${ }^{1}$ Departamento de Farmácia, ${ }^{2}$ Departamento de Estatística e Matemática Aplicada, ${ }^{3}$ Departamento de \\ Medicina Clínica, Universidade Federal do Ceará, Fortaleza, CE, Brasil
}

Correspondence to: P.F.C. de Bruin, Departamento de Medicina Clínica, Universidade Federal do Ceará, Rua Prof. Costa Mendes, 1608, $4^{\circ}$ andar, 60430-040 Fortaleza, CE, Brasil

E-mail: pfelipe@superig.com.br

\begin{abstract}
Disturbed sleep is common in chronic obstructive pulmonary disease (COPD). Conventional hypnotics worsen nocturnal hypoxemia and, in severe cases, can lead to respiratory failure. Exogenous melatonin has somnogenic properties in normal subjects and can improve sleep in several clinical conditions. This randomized, double-blind, placebo-controlled study was carried out to determine the effects of melatonin on sleep in COPD. Thirty consecutive patients with moderate to very severe COPD were initially recruited for the study. None of the participants had a history of disease exacerbation 4 weeks prior to the study, obstructive sleep apnea, mental disorders, current use of oral steroids, methylxanthines or hypnotic-sedative medication, nocturnal oxygen therapy, and shift work. Patients received $3 \mathrm{mg}$ melatonin $(N=12)$ or placebo $(N=13)$, orally in a single dose, $1 \mathrm{~h}$ before bedtime for 21 consecutive days. Sleep quality was assessed by the Pittsburgh Sleep Quality Index (PSQI) and daytime sleepiness was measured by the Epworth Sleepiness Scale. Pulmonary function and functional exercise level were assessed by spirometry and the 6-min walk test, respectively. Twenty-five patients completed the study protocol and were included in the final analysis. Melatonin treatment significantly improved global PSQI scores $(P=0.012)$, particularly sleep latency $(P=0.008)$ and sleep duration $(P=0.046)$. No differences in daytime sleepiness, lung function and functional exercise level were observed. We conclude that melatonin can improve sleep in COPD. Further long-term studies involving larger number of patients are needed before melatonin can be safely recommended for the management of sleep disturbances in these patients.
\end{abstract}

Key words: Chronic obstructive pulmonary disease; Melatonin; Sleep

Presented at the XI Congresso Brasileiro do Sono, Fortaleza, CE, Brazil, November 11-14, 2007.

Received May 10, 2008. Accepted October 14, 2008

\section{Introduction}

Chronic obstructive pulmonary disease (COPD) is a debilitating respiratory condition characterized by progressive airflow limitation primarily caused by cigarette smoking. It represents a major cause of morbidity and mortality worldwide (1-3).

Poor-quality sleep is a common complaint in COPD. Objective evidence of disturbed sleep, including reduced sleep efficiency, delayed sleep onset, reduced total sleep time, and frequent periods of wakefulness, has been well documented $(4,5)$. Impaired sleep in COPD is probably a consequence of multiple factors including nocturnal cough, nocturnal dyspnea, use of medication, and the effects of ageing and comorbidities. Hypoxemia is also believed to be a determinant of disturbed sleep in COPD (6). Sleep problems have been associated with daytime symptoms and chronic fatigue commonly found in COPD (7). Although the potential effect of chronic sleep disturbance on lung function in this disease is unknown, small transient reductions in forced vital capacity (FVC) and forced expiratory volume in $1 \mathrm{~s}$ (FEV1) have been described follow- 
ing one night's sleep deprivation in COPD patients (8). Conventional hypnotics should not be used in hypercapnic patients with COPD because they may precipitate respiratory failure due to further inhibition of ventilatory responses. In normocapnic patients, benzodiazepines have been reported to increase frequency and duration of nocturnal hypoxemia (9).

The pineal hormone melatonin is the neuroendocrine transducer of the light-dark cycle. It plays an important role in the regulation of human circadian rhythms and may have sleep-inducing activity in humans (10). There is also considerable evidence that melatonin has immunomodulatory and anti-oxidant properties (11). Exogenous melatonin administration has been shown to improve sleep in several medical conditions, including asthma $(12,13)$, although reported effects have been variable $(14,15)$. The safety of melatonin treatment up to at least 3 months has been confirmed by a recent meta-analysis (15).

The aim of the present study was to evaluate the effect of 21 days of melatonin administration on subjective sleep quality in ambulatory patients with clinically stable COPD.

\section{Subjects and Methods}

\section{Subjects}

Thirty consecutive patients regularly attending the Respiratory Outpatient Clinic at the University Hospital of the Federal University of Ceará, Brazil, were initially recruited for the study. Individuals were eligible to participate if they were 40 years of age or older, had a medical diagnosis of stage II to IV COPD according to the Global Initiative for Chronic Obstructive Lung Disease (GOLD) criteria (1) and were considered to be in a clinically stable condition by the attending physician. Exclusion criteria were disease exacerbation 4 weeks prior to the study, presence of obstructive sleep apnea or mental disorders, current use of oral steroids, methylxanthines or hypnotic-sedative medication, nocturnal oxygen therapy, and shift work. Twenty-five patients completed the study protocol and were included in the final analysis. The study protocol was approved by the local Research Ethics Committee and written informed consent was obtained from all patients.

\section{Study design}

This was a randomized, double-blind, parallel-group, placebo-controlled study of individuals with COPD. Initially, quality of sleep and daytime sleepiness were assessed by the Pittsburgh Sleep Quality Index (PSQI) and the Epworth Sleepiness Scale (ESS), respectively; lung function was evaluated by spirometry, and functional exercise level was measured by the 6-min walk test. Patients were asked to indicate their habitual in- and out-of-bed time, during the previous 5 days. They were also instructed to keep a diary with records of their in- and out-ofbed times during the 21-day study period. Patients were then randomized to the melatonin or placebo group. Fast-release $3 \mathrm{mg}$ melatonin (Natural Wealth, USA) or placebo was supplied in identical capsules to be taken in a single dose $1 \mathrm{~h}$ before bedtime for 21 consecutive days. Other medications were maintained as prescribed by the attending physician. Subjects were not required to keep any specific sleep-wake schedule. Assessment of sleep quality, daytime sleepiness, lung function and functional exercise level was repeated at the end of the treatment for comparison. All measurements were performed between 9 and 12 am. In- and out-of-bed times for the last 5 days of treatment were averaged for each group and compared with pre-treatment values. Subjects were contacted by telephone at least once a week to check for adverse effects and compliance. Patients and investigators were unaware of treatment allocation at all times.

\section{Outcomes}

The primary outcome measure was quality of sleep assessed by the PSQI. The PSQI is a seven-component scale, each one dealing with a major aspect of sleep: 1) subjective sleep quality; 2) sleep latency; 3) sleep duration; 4) sleep efficiency; 5) sleep disturbance; 6) use of sleep medication, and 7) daytime dysfunction. These components are weighted equally on a 0-3 scale, with a global score ranging from 0-21. A global PSQI score above five has been found to have a sensitivity of $89.6 \%$ and specificity of $86.5 \%$ in differentiating good from poor sleepers (16). Component number 6 always scored zero because individuals on sleep medications were not included in this study.

Secondary outcome measures were daytime somnolence, functional exercise level and lung function. Daytime somnolence was assessed by the ESS, a questionnaire that measures a subject's expectation of dozing in eight hypothetical situations. Dozing probability ratings range from zero (none) to three (high probability). A total score of 10 or more is considered to be indicative of excessive daytime sleepiness (17).

Functional exercise capacity was evaluated by the 6min walk test. This is a practical simple test that measures the distance a patient can quickly walk on a flat, hard surface in a period of $6 \mathrm{~min}$, the 6 -min walk distance (6MWD). The test was completed in an enclosed corridor on a flat course $20 \mathrm{~m}$ in length. In brief, patients were asked to walk at their highest rate from one end of the corridor to the other end as many times as possible within 
the established time of $6 \mathrm{~min}$. The test was performed under the control of one of the investigators who encouraged the patients. At the end of $6 \mathrm{~min}$, the investigator measured the distance walked by the patient (18).

Spirometry was performed according to recommended techniques. Measurements included FEV1, FVC, and FEV1/FVC ratio and results were compared with previously published normal values (19).

\section{Statistical analysis}

Data were examined for normality using the ShapiroWilk test. Between-group comparisons of age and body mass index were made with the unpaired Student $t$-test. Past cigarette consumption in both groups was compared by the Mann-Whitney test. ESS scores, spirometric results, 6MWD, and in- and out-of-bed times, as well as within-group comparisons (before and after treatment) of the same variables of the melatonin and placebo groups were compared with two-way ANOVA. Differences in global PSQI scores as well as in PSQI component scores before and after treatment were analyzed for each group using the Wilcoxon test. Between-group comparisons of global PSQI scores and PSQI component scores were made with the Mann-Whitney test. Additionally, patients were divided into two categories: good sleepers (PSQI $\leq 5$ ) and poor sleepers (PSQI >5). Distribution of good and poor sleepers in melatonin and placebo groups were compared using the Fisher exact test. Within-group comparisons of good and poor sleepers (before and after treatment) were made using the MacNemar test. Statistical analysis was performed with the Statistical Package for Social Sciences (20). Data are reported as mean $\pm S D$ and the level of significance was set at $P<0.05$.

\section{Results}

Thirty patients with stage II to IV COPD were initially recruited for the study. Five individuals ( 2 from the melatonin group and 3 from the placebo group) were removed from the study because of an exacerbation of COPD (2 patients), hospitalization for non-respiratory reasons (1 patient), alleged adverse effects of treatment (1 patient), and loss of follow-up (1 patient). Demographic and clinical characteristics of the 25 patients who finished the protocol are shown in Table 1. No significant differences were found between the two groups with respect to age, smoking history, body mass index, global PSQI scores, in- and outof-bed times, ESS score, 6MWD, FEV1 \% predicted and FEV1/FVC ratio before treatment $(P>0.05)$. Mean FVC \% predicted was lower in the placebo group compared with the melatonin group before treatment $(P=0.020)$.

\section{Outcome measures}

On initial assessment, 16 patients ( 8 from the melato-

Table 1. Demographic characteristics, quality of sleep, daytime sleepiness, pulmonary function, and functional exercise level before and after treatment with melatonin or placebo.

\begin{tabular}{lccccc}
\hline & \multicolumn{2}{c}{ Melatonin $(\mathrm{N}=12)$} & & \multicolumn{2}{c}{ Placebo $(\mathrm{N}=13)$} \\
\cline { 2 - 3 } \cline { 5 - 6 } & Before & After & & Before & After \\
\hline Gender (male/female) & $7 / 5$ & & & $7 / 6$ & \\
Age (years) & $64.17 \pm 9.9$ & & & $67.38 \pm 8.1$ & \\
Smoking history (pack-years) & $42.87 \pm 25.8$ & & & $44.92 \pm 29.9$ & \\
BMI (kg/m $)$ & $23.87 \pm 4.2$ & $23.80 \pm 4.2$ & & $24.10 \pm 4.0$ & $24.14 \pm 4.0$ \\
PSQI & $7.75 \pm 4.0^{*}$ & $4.58 \pm 1.2^{*}$ & & $6.38 \pm 3.0$ & $5.54 \pm 2.2$ \\
Good/poor sleepers & $4 / 8^{* * *}$ & $11 / 1^{* * *}$ & & $5 / 8$ & $7 / 6$ \\
In-bed time (decimal hours) & $22.65 \pm 0.94$ & $22.36 \pm 1.31$ & & $22.20 \pm 1.05$ & $22.48 \pm 0.83$ \\
Out-of-bed time (decimal hours) & $5.25 \pm 0.82$ & $5.43 \pm 0.92$ & & $5.43 \pm 1.37$ & $5.57 \pm 0.88$ \\
ESS & $7.83 \pm 5.5$ & $7.17 \pm 5.1$ & & $7.31 \pm 3.9$ & $8.08 \pm 4.9$ \\
FVC (\% predicted) & $83.67 \pm 12.2^{* *}$ & $83.02 \pm 18.5$ & & $68.72 \pm 16.3^{* *}$ & $71.64 \pm 15.3$ \\
FEV1 (\% predicted) & $57.52 \pm 14.0$ & $56.81 \pm 19.2$ & & $46.78 \pm 17.0$ & $48.83 \pm 17.3$ \\
FEV1/FVC ratio & $54.97 \pm 8.7$ & $54.26 \pm 10.3$ & & $52.83 \pm 10.5$ & $52.96 \pm 10.6$ \\
6MWD (m) & $356.3 \pm 70.0$ & $361.6 \pm 58.6$ & & $332.8 \pm 67.0$ & $340.7 \pm 55.4$
\end{tabular}

Data are reported as means \pm SD. BMI = body mass index; PSQI = Pittsburgh Sleep Quality Index; ESS = Epworth Sleepiness Scale; FVC = forced vital capacity; FEV1 = forced expiratory volume in $1 \mathrm{~s} ; 6 \mathrm{MWD}=6$-min walk distance. ${ }^{*} P=0.012$, comparison of before and after melatonin treatment (Wilcoxon test); ${ }^{* *} P=0.020$, comparison of before treatment with melatonin and placebo (ANOVA); ${ }^{\star \star *} \mathrm{P}=0.016$, comparison of before and after melatonin treatment (MacNemar test). 
nin group) were classified as poor sleepers. A significant improvement in the relative frequency of good $v s$ poor sleepers was observed after melatonin treatment $(P=$ $0.016)$, but not after placebo $(P>0.05)$. A trend toward an improvement in the distribution of good vs poor sleepers was detected in the group treated with melatonin compared with placebo $(P=0.073)$. Global $P S Q I$ score was significantly improved by melatonin treatment $(P=0.012$; Table 1). Global PSQI scores before and after treatment for individuals in both groups are shown in Figure 1. A significant reduction in the PSQI component sleep latency $(P=0.008)$, an increase in sleep duration $(P=0.046)$ and a trend toward an improvement in sleep efficiency $(P=$ 0.057 ) were found after melatonin treatment.

Excessive daytime sleepiness was detected in 5 subjects from the melatonin group and in 4 subjects from the placebo group. Treatment with melatonin or placebo did not significantly change ESS scores. No significant differences were observed in lung function parameters or in the $6 \mathrm{MWD}$ after treatment with melatonin or placebo (Table 1).

\section{Adverse effects}

A subject from the placebo group left the study because of an alleged side-effect (tongue swelling). Four of the 25 cases included in the final analysis reported ad-

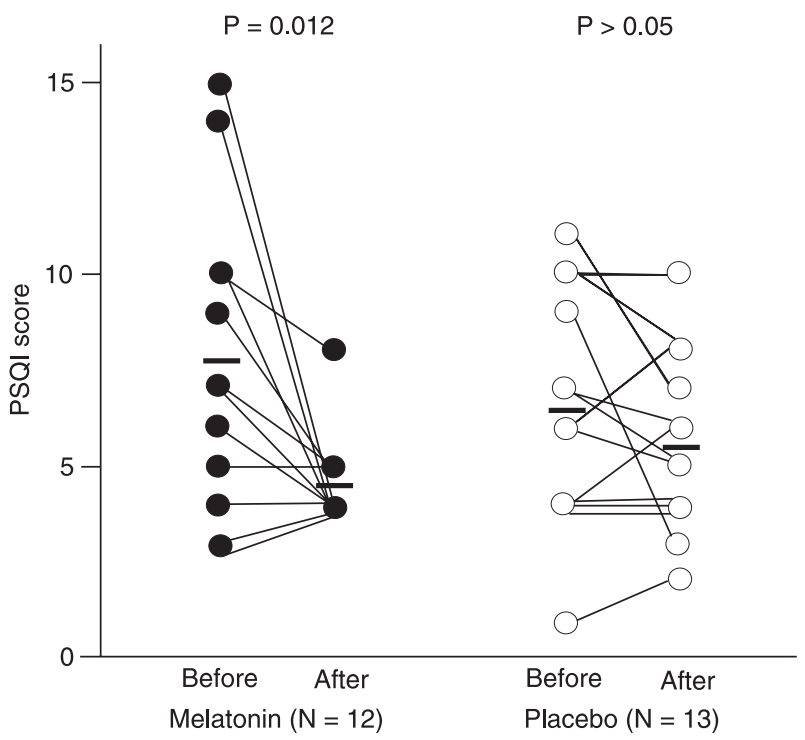

Figure 1. Melatonin, but not placebo, significantly improved subjective quality of sleep in patients with chronic obstructive pulmonary disease. The $P$ values shown are for the comparison of Pittsburgh Sleep Quality Index (PSQI) scores before and after treatment with melatonin and placebo. The mean values for both groups are shown as thick horizontal lines. The statistical test used was the Wilcoxon test. verse effects: mild headache (1 subject from the melatonin group); dryness of the mouth (1 subject from the melatonin group and 1 from the placebo group) and loss of appetite (1 subject from the placebo group).

\section{Discussion}

The results of this study show that $3 \mathrm{mg}$ melatonin taken $1 \mathrm{~h}$ before bedtime can improve sleep in patients with moderate to very severe COPD. To our knowledge, this is the first study on melatonin and sleep in ambulatory patients with COPD. Previously, Shilo and co-workers (21) conducted a pilot study on the effects of exogenous melatonin on sleep quality in 8 adult patients hospitalized in the pulmonary intensive care unit with respiratory failure caused by exacerbation of COPD or with pneumonia. Patients received either $3 \mathrm{mg}$ of controlled-release melatonin or placebo at $10 \mathrm{pm}$. Sleep quality was evaluated by actigraphy. Treatment with melatonin improved both the duration and quality of sleep.

It is generally accepted that melatonin administered during the day, when its endogenous levels are low, has somnogenic properties $(10,22)$. When used near the time of habitual sleep, melatonin has produced less consistent results. Nocturnal administration has been reported to promote sleep $(23,24)$, to have no significant clinical benefit (25) or to even increase wake time after sleep onset (26). Such variability may be related to differences in inclusion and exclusion criteria, outcome measures, hormone dose, and routes and timing of its administration. A recent metaanalysis based on data derived from 17 studies indicates that melatonin can produce small but significant benefits in sleep onset latency, sleep efficiency and sleep duration (14). Importantly, such improvements seem to be associated with better performance in tests of attention, concentration, fine motor activity, and reaction time and with the sensation of improved daytime well being (27). In addition to its sleep-inducing effects, melatonin has chronobiotic properties, i.e., the ability to phase-shift the human circadian system by advance or delay according to a phaseresponse curve or to re-entrain a desynchronized circadian rhythm (28). Both the sleep-inducing and the circadian effect may provide useful tools for improving sleep in COPD and other similar clinical conditions.

The mechanism for melatonin sleep-inducing effect has not been fully elucidated. Involvement of a GABAergic mechanism has been suggested by experimental evidence of melatonin interaction with central benzodiazepine receptors (29). However, a clinical trial with flumazenil, a benzodiazepine antagonist, failed to demonstrate a decrease in hypnotic and hypothermic effects 
of melatonin (30). Plasma cGMP shows circadian variation, with peak levels being observed during nocturnal sleep. Melatonin administration promotes an increase in cGMP, with peak levels coinciding with melatonin acrophase. Moreover, peak plasma levels of melatonin and cGMP show a positive correlation with sleepiness, pointing to an involvement of cGMP in melatonin hypnotic action (31). It has also been suggested that melatonin may act to facilitate sleep by inhibiting the circadian drive for waking that comes from the suprachiasmatic nucleus (10). This is supported by evidence from studies in both day-active animals (32) and humans (33) that the circadian pacemaker promotes wakefulness at certain times of day, together with evidence that neuronal firing of the mammalian suprachiasmatic nucleus is inhibited by suprachiasmatic nucleus $\mathrm{Mel}_{1 \mathrm{a}}$ receptor-specific melatonin binding (34).

On average, the subjects in the present study did not present daytime somnolence. The scarce literature published on daytime sleepiness in patients with COPD is conflicting. Cormick et al. (35) evaluated sleep quality and daytime sleepiness in fifty patients with COPD and reported difficulty in getting to sleep and staying asleep and daytime sleepiness compared with age-matched controls without symptomatic lung disease. Interestingly, more than twice as many patients $(28 \%)$ as controls (10\%) reported regular use of hypnotics. Orr and co-workers (36) studied 14 patients with COPD and chronic hypoxemia and were unable to find any evidence of daytime sleepiness, despite polysomnographic demonstration of a short sleep time and an increased number of arousals from sleep. In the present study, improved sleep after melatonin treatment was not accompanied by any significant change in sleepiness scores. It has been recognized that, in contrast to common hypnotics, melatonin does not produce a rapid increase in subjective sleepiness or major impairments in cognitive performance (37). A study comparing the effects of melatonin and temazepam on sleepiness and cognitive performance showed that while temazepam causes an abrupt but short-lived increase in subjective sleepiness, melatonin gradually increases sleepiness. A smaller deficit in performance on a range of neurobehavioral tasks was observed after melatonin administration compared with temazepam (38).

\section{References}

1. Rabe KF, Hurd S, Anzueto A, Barnes PJ, Buist SA, Calverley $P$, et al. Global strategy for the diagnosis, management, and prevention of chronic obstructive pulmonary disease: GOLD executive summary. Am J Respir Crit Care Med 2007; 176:
We studied patients predominantly with severe COPD. On initial assessment, a lower FVC was found for the placebo group compared to the melatonin group, suggesting a higher degree of lung hyperinflation and mechanical impairment in the placebo group. Although disturbed sleep in COPD is thought to be related to the underlying disease process (6), no significant difference in sleep quality between the two groups was found at baseline, as mentioned before. Improved quality of sleep associated with melatonin treatment could not be correlated with any improvement in daytime lung function or functional exercise level.

No significant adverse effects were reported by the subjects after melatonin administration. Melatonin has been consumed for many years in several countries where it is available freely or by prescription, without reports of major adverse effects. Recently, a systematic review of 17 randomized controlled trials with 651 participants confirmed that melatonin is safe for short-term use (three months or less). However, there is a need for further studies assessing the safety of long-term melatonin treatment (15). In particular, there has been some concern regarding the effect of prolonged melatonin use on the human reproductive function. A decrease in sperm concentration and motility below normal was found in 2 of 8 men enrolled in a double-blind crossover study of melatonin treatment (39).

In the present study, no objective measures of sleep or daytime sleepiness were obtained. Sleep quality was assessed by the PSQI, a questionnaire widely used for this purpose. Previously, melatonin has been shown to improve subjective sleep quality in the absence of substantial changes in sleep architecture $(13,14)$. It should be kept in mind that, due to its largely subjective nature, sleep quality correlates with, but is not accurately defined by, sleep laboratory measurements. A previous study comparing subjective sleep characteristics assessed by questionnaire with objective measures obtained during polysomnography showed that, although individuals are able to satisfactorily judge time in bed, total sleep time and sleep latency, correlation between sleep quality and objective sleep characteristics is less strong (40). In summary, melatonin, as used in this study, has the potential to become a useful tool for the management of sleep problems in COPD patients.

532-555.

2. Mannino DM, Holguin F. Epidemiology and global impact of chronic obstructive pulmonary disease. Respir Med: COPD Update 2006; 1: 114-120. 
3. Menezes AM, Perez-Padilla R, Jardim JR, Muino A, Lopez MV, Valdivia G, et al. Chronic obstructive pulmonary disease in five Latin American cities (the PLATINO study): a prevalence study. Lancet 2005; 366: 1875-1881.

4. Weitzenblum E, Chaouat A. Sleep and chronic obstructive pulmonary disease. Sleep Med Rev 2004; 8: 281-294.

5. Santos CEVG, Viegas CAA. Sleep pattern in patients with chronic obstructive pulmonary disease and correlation among gasometric, spirometric, and polysomnographic variables. J Pneumol 2003; 29: 69-74.

6. McNicholas WT. Sleep in chronic obstructive pulmonary disease. Eur Respir Mon 2006; 38: 325-336. [http:// www.maney.co.uk/series/ers]

7. Breslin E, van der Schans C, Breukink S, Meek P, Mercer K, Volz W, et al. Perception of fatigue and quality of life in patients with COPD. Chest 1998; 114: 958-964.

8. Phillips BA, Cooper KR, Burke TV. The effect of sleep loss on breathing in chronic obstructive pulmonary disease. Chest 1987; 91: 29-32.

9. Kutty K. Sleep and chronic obstructive pulmonary disease. Curr Opin Pulm Med 2004; 10: 104-112.

10. Scheer FA, Czeisler CA. Melatonin, sleep, and circadian rhythms. Sleep Med Rev 2005; 9: 5-9.

11. Claustrat B, Brun J, Chazot G. The basic physiology and pathophysiology of melatonin. Sleep Med Rev 2005; 9: 11-24.

12. Campos FL, da Silva-Junior FP, de Bruin V, de Bruin PF. Melatonin improves sleep in asthma: a randomized, doubleblind, placebo-controlled study. Am J Respir Crit Care Med 2004; 170: 947-951.

13. Medeiros CA, Carvalhedo de Bruin PF, Lopes LA, Magalhaes MC, de Lourdes SM, de Bruin V. Effect of exogenous melatonin on sleep and motor dysfunction in Parkinson's disease. A randomized, double blind, placebo-controlled study. J Neurol 2007; 254: 459-464

14. Brzezinski A, Vangel MG, Wurtman RJ, Norrie G, Zhdanova I, Ben-Shushan A, et al. Effects of exogenous melatonin on sleep: a meta-analysis. Sleep Med Rev 2005; 9: 41-50.

15. Buscemi N, Vandermeer B, Hooton N, Pandya R, Tjosvold L, Hartling L, et al. Efficacy and safety of exogenous melatonin for secondary sleep disorders and sleep disorders accompanying sleep restriction: meta-analysis. BMJ 2006; 332: $385-393$

16. Buysse DJ, Reynolds CF III, Monk TH, Berman SR, Kupfer DJ. The Pittsburgh Sleep Quality Index: a new instrument for psychiatric practice and research. Psychiatry Res 1989; 28: $193-213$

17. Johns MW. A new method for measuring daytime sleepiness: the Epworth Sleepiness Scale. Sleep 1991; 14: 540-545.

18. ATS statement: guidelines for the six-minute walk test. $A m \mathrm{~J}$ Respir Crit Care Med 2002; 166: 111-117.

19. American Thoracic Society. Standardization of spirometry, 1994 update. Am J Respir Crit Care Med 1995; 152: 1107-1136.

20. Norusis MJ. SPSS for Windows: Base System User's Guide. Release 15.0. [Computer program]. 2006.

21. Shilo L, Dagan Y, Smorjik Y, Weinberg U, Dolev S, Komptel B, et al. Effect of melatonin on sleep quality of COPD intensive care patients: a pilot study. Chronobiol Int 2000; 17: 71-76.

22. Pires ML, Benedito-Silva AA, Pinto L, Souza L, Vismari L, Calil HM. Acute effects of low doses of melatonin on the sleep of young healthy subjects. J Pineal Res 2001; 31: 326-332.

23. Zhdanova IV, Wurtman RJ, Lynch HJ, Ives JR, Dollins AB,
Morabito C, et al. Sleep-inducing effects of low doses of melatonin ingested in the evening. Clin Pharmacol Ther 1995; 57: 552-558.

24. Cajochen C, Krauchi K, Danilenko KV, Wirz-Justice A. Evening administration of melatonin and bright light: interactions on the EEG during sleep and wakefulness. J Sleep Res 1998; 7: 145-157.

25. Hughes RJ, Sack RL, Lewy AJ. The role of melatonin and circadian phase in age-related sleep-maintenance insomnia: assessment in a clinical trial of melatonin replacement. Sleep 1998; 21: 52-68.

26. Dawson D, Rogers NL, van den Heuvel CJ, Kennaway DJ, Lushington K. Effect of sustained nocturnal transbuccal melatonin administration on sleep and temperature in elderly insomniacs. J Biol Rhythms 1998; 13: 532-538.

27. Waldhauser F, Saletu B, Trinchard-Lugan I. Sleep laboratory investigations on hypnotic properties of melatonin. Psychopharmacology 1990; 100: 222-226.

28. Arendt J. Importance and relevance of melatonin to human biological rhythms. J Neuroendocrinol 2003; 15: 427-431.

29. Tenn CC, Niles LP. Central-type benzodiazepine receptors mediate the antidopaminergic effect of clonazepam and melatonin in 6-hydroxydopamine lesioned rats: involvement of a GABAergic mechanism. J Pharmacol Exp Ther 1995; 274: 84-89.

30. Nave R, Herer P, Haimov I, Shlitner A, Lavie P. Hypnotic and hypothermic effects of melatonin on daytime sleep in humans: lack of antagonism by flumazenil. Neurosci Lett 1996; 214: 123-126.

31. Zhdanova IV, Raz DJ. Effects of melatonin ingestion on cAMP and cGMP levels in human plasma. J Endocrinol 1999; 163: 457-462.

32. Edgar DM, Dement WC, Fuller CA. Effect of SCN lesions on sleep in squirrel monkeys: evidence for opponent processes in sleep-wake regulation. J Neurosci 1993; 13: 1065-1079.

33. Dijk DJ, Czeisler CA. Paradoxical timing of the circadian rhythm of sleep propensity serves to consolidate sleep and wakefulness in humans. Neurosci Lett 1994; 166: 63-68.

34. Liu C, Weaver DR, Jin X, Shearman LP, PieschI RL, Gribkoff VK, et al. Molecular dissection of two distinct actions of melatonin on the suprachiasmatic circadian clock. Neuron 1997; 19: 91-102.

35. Cormick W, Olson LG, Hensley MJ, Saunders NA. Nocturnal hypoxaemia and quality of sleep in patients with chronic obstructive lung disease. Thorax 1986; 41: 846-854.

36. Orr WC, Shamma-Othman Z, Levin D, Othman J, Rundell $\mathrm{OH}$. Persistent hypoxemia and excessive daytime sleepiness in chronic obstructive pulmonary disease (COPD). Chest 1990; 97: 583-585.

37. Zhdanova IV. Melatonin as a hypnotic: pro. Sleep Med Rev 2005; 9: 51-65.

38. Rogers NL, Kennaway DJ, Dawson D. Neurobehavioural performance effects of daytime melatonin and temazepam administration. J Sleep Res 2003; 12: 207-212.

39. Luboshitzky R, Shen-Orr Z, Nave R, Lavi S, Lavie P. Melatonin administration alters semen quality in healthy men. $J$ Androl 2002; 23: 572-578.

40. Armitage R, Trivedi M, Hoffmann R, Rush AJ. Relationship between objective and subjective sleep measures in depressed patients and healthy controls. Depress Anxiety 1997; 5: 97-102 